\title{
In Vitro Fertilization Genetic Algorithm
}

\author{
Celso G. Camilo-Junior ${ }^{1}$ and Keiji Yamanaka ${ }^{2}$ \\ ${ }^{1}$ Informatics Institute, Federal University of Goias \\ ${ }^{2}$ School of Electrical Engineering, Federal University of Uberlandia \\ Brazil
}

\section{Introduction}

Some techniques are applied to the optimization problems. However, a few achieve satisfactory performance when the problem is complex, for example, multimodal or multiobjective. The Mathematical Programming algorithms which use the gradient as a search guide have difficulty and do not often reach the optimum in multimodal problems. The metaheuristics, on the other hand, don't ensure the global optimum but they have good results and, therefore, are quite used in these scenarios.

Metaheuristics could be classified by the number of potential solutions that are used: solution-based metaheuristics, such as Hill-Climbing, Simulated Annealing and Tabu search; population-based metaheuristics, such as the Evolutionary Algorithms; or hybrids metaheuristics. The population-based algorithms start the search with several points in the search space and, through the interactions of among points, try to find a new point with the highest value of the objective function. This strategy, therefore, explores the search space in several places simultaneously (Oriented Exploration). The solution-based algorithms are based on a single point to exploit the search space (Oriented Exploitation). They often use techniques to escape from the local optimum. The hybrid strategy tries to join the intensification and diversification to improve both quality of computed solutions and the robustness of solvers. All strategies show good results depending on the approached problem. As an example of metaheuristic, we can cite: Simulated Anneling, Tabu Search, Grasp, VND, VNS and Ant Colony. Among them, Evolutionary Algorithms, especially the Genetic Algorithms, show excellent results and thus they are one of the most popular among researchers.

Genetic algorithms are optimization methods inspired by the mechanisms of alive beings' evolution Goldberg (1989). The algorithms based on this technique follow the principle of natural selection and survival of the fittest (Charles Darwin).

One of the genetic algorithm advantages is the simplification in the formulation and solution of optimization problems. Simple GAs usually work with coded descriptions formed by bits strings of fixed size. Other types of GAs can work with bits strings of variable size, e.g. GAs used for Genetic Programming FERREIRA (2001a;b); RODRIGUES (2003).

The GA is indicated for the solution of complex optimization problems, such as the real problems, that involve a large number of variables and, consequently, high dimensional search spaces. Moreover, in many cases where other optimization strategies fail in finding a solution, GAs are good options. However, in some cases, the performance is not satisfactory GEN \& CHENG (1997); MICHALEWICZ (1996). Therefore, some works are developed with the aim to improve the performance CHAINATE \& PONGCHAROEN (2007); MUSIL et al. 
(1999); PARK et al. (2000); RAJAN et al. (2002); RONG-LONG \& KOZO (2005); RUTTKAY et al. (1995); WU et al. (2004); YANG \& DOUGLAS (1998); YEN et al. (1998). Some works focus in the convergence speed, though, most analyzes the efficacy. The preservation of genetic diversity to avoid premature convergence is a frequently researched topic MAHFOUD (1992; 1995); SHIMODAIRA (2002); TACKETT \& CARMI (1994).

Thus, in this paper we propose the In Vitro Fertilization Module (IVFm), to assist the GAs evolution, avoiding the loss of information during evolution. The IVFm is an assistant algorithm that runs in a parallel flow of GA's flow and that recombines chromosomes in order to do the information mining with individuals, which are proceeding from the populations created by GAs or generated by the IVFm operators.

To test the efficacy, defined here as the capacity to reach the global optimum, and the efficiency, defined as the capacity to accelerate the GA evolution, we opted for a benchmark minimization problem that is multimodal with several local optimum. This problem was used to establish the test scenarios.

The results from these three experiments show that the IVFm, especially with the operator EAR-N, has excellent performance and contributes substantially to quick convergence of GA to the global optimum.

The chapter will be organized as follows. One section about canonical GA. One section about the IVFm and operators are described. One section about the premature convergence. One section about the experimental results. Finally, the conclusion and suggestions future works.

\section{IVFm: In Vitro Fertilization module}

The GAs evolutionary process is composed by a cyclical flow, in which each iteration creates a new generation. For each new generation, individuals are generated and introduced into the population to replace some of the existing ones, which are discarded. However, many of these eliminated individuals contain genes with information that is important for the search. Nevertheless, they do not even go through the reproduction process before being discarded. In other words, they were generated and eliminated and did not contribute to the evolution. Therefore, we can say that in every iteration some information is lost, discarded without analysis.

Although this process mimics the evolution of species, in which an individual could be born and die without generating progeny, it is well-known that there is some loss of information. The information lost may assume two possibilities. It might not go back to the population, causing in some cases a reduction in efficacy and characterizing the loss of opportunity, or it might return by mutation process with low probability. On last case, the efficiency is lower because is needed much time until that information returns and is processed. Therefore, we propose the In Vitro Fertilization Module (IVFm) that recombines the chromosomes from population of GA and new individuals to better exploit the information presented.

Assisted Reproduction is a set of techniques of reproduction where the generation of a life is manipulated and assisted by experts in order to ensure the success of the process. In Vitro Fertilization (IVF) is one of Assisted Reproduction technique that collects egg cells from the ovary of the mother and fertilizes them with prepared sperm from the father, forming the pre-embryos. After manipulation, two pre-embryos are selected and transferred to the mother. The first human baby generated by IVF born in 1978. From 1978 until today, many people have been generated by the IVF.

Analogous to IVF the IVFm assesses various combinations and selects the individual according its quality. IVFm is an algorithm executed in a parallel flow, which receives as input a portion of the GA population and as output it returns an individual, which may be better 


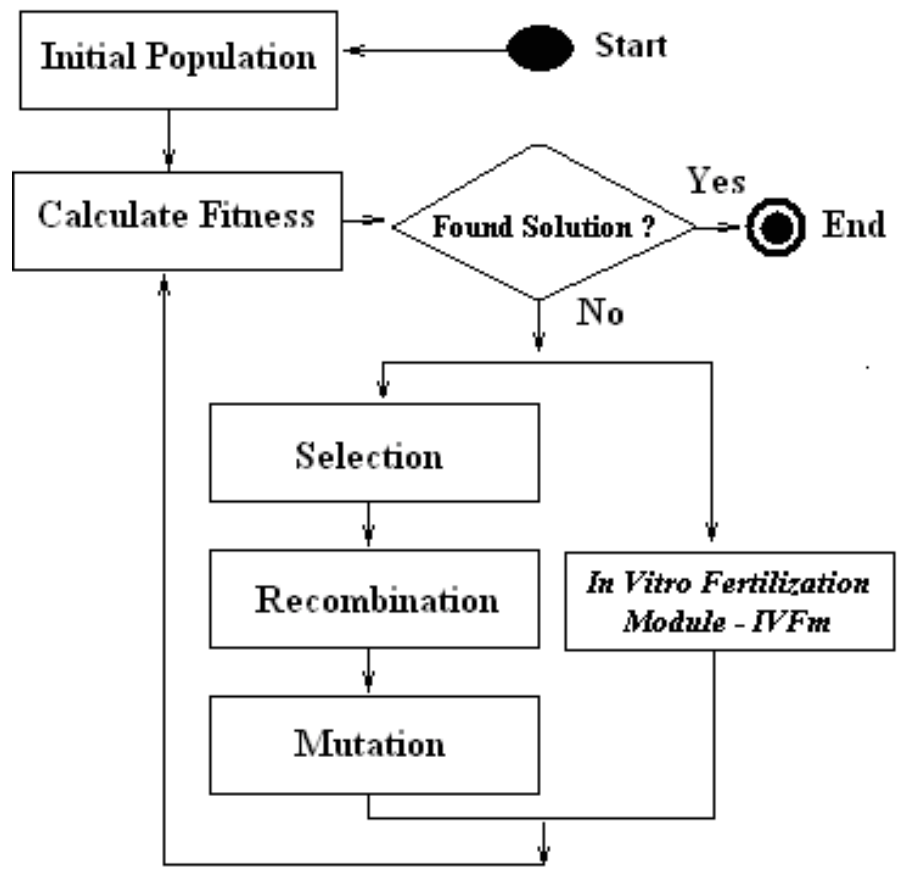

Fig. 1. GA with the IVFm

than the best current (Figure 1). Thus, the IVFm could supply the GA of good individuals, improving and accelerating the GA evolutionary process.

The ideal way to absorb all the information in individuals would be to recombine them gene-gene, until you find the best combination, which would be computationally unfeasible, especially for larger chromosomes. Therefore, we opted to generate children from the recombination of chromosome parts of some individuals with the best one (section 2.4). If the process generates a better individual, this replaces the current best. Otherwise, if the operator does not produce a better individual, there is no interference in the population.

There are two groups of operators for IVFm until this moment. The first one with operators that use as genetic material only the population generated by GA and the second one with operators that alter chromosomes that will be recombined. The second group strategy is to improve the IVFm's population with information that may be beneficial for the recombination process. The operator AR (Assisted Recombination) is a member of the first group and the operators EAR-T, EAR-P and EAR-N are members of the second group.

The IVFm is detailed in the following sections. Section 2.1 describes the execution flow, section 2.2 describes the division of genetic material, section 2.3 shows the operators and section 2.4 describes the process of recombination.

\subsection{The IVFm execution flow}

At the beginning of the GA execution, the division of the genetic material is defined (section 2.2) as well as the number of individuals that will be used by IVFm (NuIndiv). This configuration is only done in the beginning of the execution of the algorithm. 


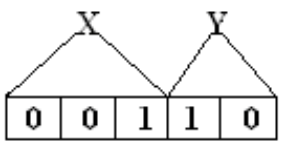

Fig. 2. Two groups selected as the genetic material to exchange

After receiving the current population of GA, each IVFm generation executes the following tasks:

1. Find the fittest individual and label it as Father;

2. Receive as input the following parameters: the N GA individuals (NuIndiv), which are handled in the recombination process;

3. On the EAR strategy, from $\mathrm{N}$ selected individuals, the half $(\mathrm{N} / 2)$ changes the chromosomes, forming $\mathrm{N}^{\prime}$. On the AR strategy $\mathrm{N}^{\prime}=\mathrm{N}$;

4. The fittest (father) and the $\mathrm{N}^{\prime}$ individuals are recombined. As there is only one father for each recombination process, the generated children are siblings;

5. If IVFm generates good individuals, they are inserted in the new population, replacing elected individuals, chosen by the elitism process. Without elitism, individuals replace any others aleatorily.

Task 5 above, explains the interference form of IVFm in the population, generated by GA. The interference only happens when good individuals, better than the best available previously, are generated by the proposed algorithm.

\subsection{Division of Genetic Material}

Before the recombination (exchange of genetic material) the chromosome is divided into groups of genes or gene to gene. This is called the Genetic Material Division to be exchanged. This is an important step of the algorithm, because we believe that knowledge of the problem helps the designer to choose the best way to divide the chromosome, and so, help the algorithm to solve the problem.

One option is to divide the chromosome according to the coded variables, e.g. if the first 3 genes represent the variable $x$ and the past 2 the $y$, then the chromosome is divided into two groups, the first one containing 3 genes and the other one 2 (Figure 2). Thus, in recombination process the exchanges are among the variable values (decoded values) and not among encoded values. Therefore, this process provides the designer with a tool for knowledge transferring.

\subsection{IVFm's operators}

Four independent operators are proposed for the IVFm as strategies to help the GA to explore the search space. The AR recombines the chromosomes of the current unchanged population. On the EAR-T, all the genes of some chromosomes suffer mutation before recombination process. On the EAR-P, one group of genes, formed by Genetic Material Division, suffer mutation before recombination and EAR-N generates new individuals to participate in the recombination process. All EAR operators (section 2.3.2) produce some kind of change in population before the recombination, while the AR operator (section 2.3.1) uses the current population without changes as genetic material. The operators are independent and the combined use of these has not been tested yet. 


\subsubsection{Assisted Recombination operator}

Because of the low utilization by the GA of genetic material, we propose the Assisted Recombination (AR) - an operator of the IVFm prepared to better exploit the genetic information in chromosomes of the population generated by the GA. It is hoped, therefore, that the operator contributes to genetic improvement.

The operator is composed of a single phase, called recombination (see details in section 2.4). In this phase, the operator receives part of the GA population and, without making changes to parents, produces children who are analyzed. If the operator finds better individual, this replaces the current best. If the operator does not produce better individuals, there is no interference in the population.

One of the principal AR features is the capability of assisting the recombinations and finding individuals with good fitness, sometimes better than the fittest.

\subsubsection{Exploratory Assisted Recombination operators}

The Exploratory Assisted Recombination (EAR) operators are based on Assisted Recombination and, therefore, have similarities. For instances, they use the $\mathrm{N}$ individuals of the GA population in the improvement process, they have capacity to guide the recombination and they have capacity to identify better individuals than the best current.

Like the Assisted Recombination, the aim of the EAR operators is to better exploit the information produced by GA. The difference of this new class of operators is the ability to make global search through the added exploratory characteristic.

The EAR operators have two phases. The first one, called search space exploration, changes some chromosomes received from the GA and the second one that recombines the chromosomes. These phases are the same to all operators of IVFm (section 2.4).

During the first phase (exploration of search space), the population of $\mathrm{N}$ selected individuals is divided in half $(\mathrm{N} / 2)$ and the individuals of last portion are totally (EAR-T) or partially (EAR-P) changed.

The operator EAR-P applies mutation to the raffled part of the chromosome, divided by the genetic material division. The operator EAR-T applies mutation to all the chromosomes and the EAR-N operator randomly generates new N/2 individuals. After the modifications, the changed individuals replace the $\mathrm{N} / 2$ of the population.

Figure 3 describes the process from the choice of $\mathrm{N} / 2$ individuals until the new changed population $\left(\mathrm{N}^{\prime}\right)$, which will be used during the recombination (section 2.4). Is established for this example the division of the genetic material into two parts. The first, with 3 genes and the second with 2 genes. The number of individuals from the GA is 12 (qtdIndv), so the last 6 (N / 2) are changed.

\subsection{The recombination}

After establishing the groups of genes (division of genetic material) and the population $\mathrm{N}^{\prime}$, the next step is recombination. In this phase, the chromosome is represented by a vector and every element of this is a group of genes, defined by the division of genetic material. This process is similar to the one applied in the Jung's paper Jung (2003).

Analyzing the population $\mathrm{N}^{\prime}$, the best individual is reserved as father and the others as mothers, being the number of mothers limited by the parameter NuIndiv. To create a son, the mother gives an element of its vector and the father supplements the son's chromosome with the other elements of its own vector. This process repeats for all mothers, generating a group of offspring. Other groups are generated by the exchange of the element donated by the mother and the father's chromosome complement, thus, the number of groups of offspring is equal to the number of elements of the father's vector. 


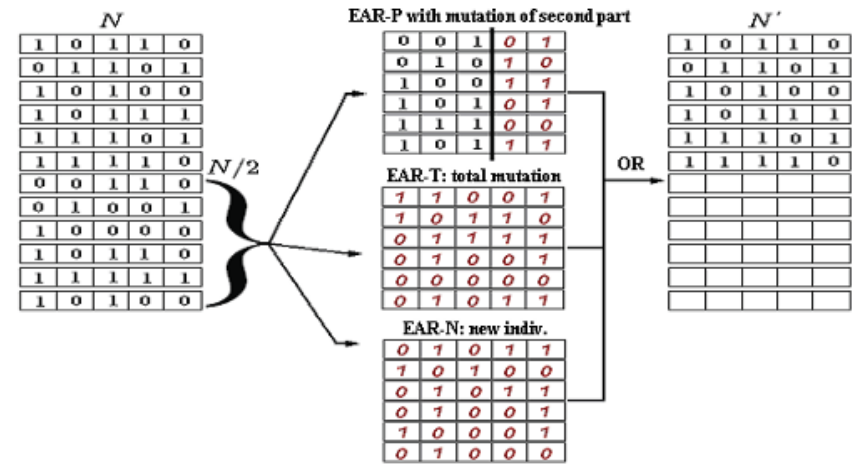

Fig. 3. EAR's exploration process

After the generation of all groups of offspring, the best child is considered the best Super Individual and it is compared to the father. If better, the son replaces the father in the IVFm's population and the recombination process restarts. If not, the algorithm interrupts the loop and inserts the current father in the GA population.

As an example of a recombination iteration, Figure 4 shows:

- The divided chromosome (division of genetic material) into two parts. Group 1 with 3 genes and group 2 with 2 genes;

- Three mothers established by the parameter NuIndiv $=3$, in which the mothers are the three individuals after the father;

- The first group of offspring generated by the donation of the first element of the mother vector and a complement of the father's. For example, the second child from the "Sons of Group 1" (01110) is the result of the union of the first element of the second mother (011) and of the father's complement, i.e. second element of the father vector (10);

- The second group of offspring. In this group, the mother donates the second vector element and the father completes it with its first vector element. For example, the third child from the "Sons of Group 2" (00100) is the result of the union of the second element of the third mother (00) and of the father's complement, i.e. first element of the father's vector (001). In this case, contrary to the "Sons of Group 1", the child is formed by the father's first part and the mother's second part;

- The best individual from the group of offspring is considered the super individual of the group. The best among the super individuals is compared to the father. If it is better than the father, the best super individual replaces the father in the next iteration. If not, the algorithm interrupts the loop and returns the current father.

\section{The premature convergence}

One of the main features of the IVFm is the ability to better exploit the information present in the population (information mining), generating good individuals who can improve and accelerate the genetic evolution.

However, when we try to accelerate the GA's genetic evolution a problem arise, the premature convergence Goldberg (1989); SULTAN et al. (2007), defined as a fast loss of genetic diversity in the population that harms the final solution. Despite being considered a problem when 
Start

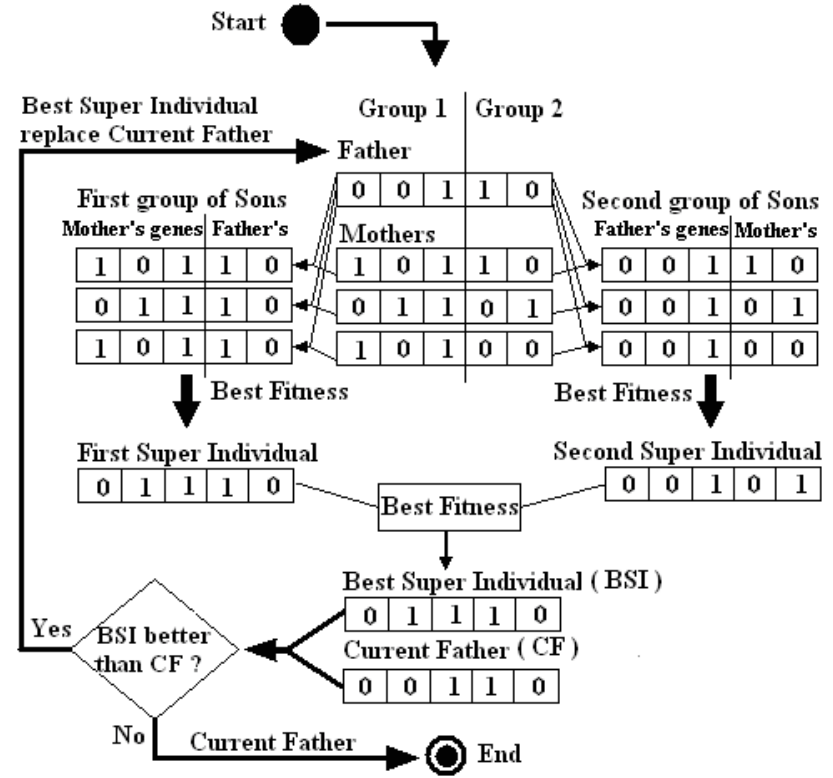

Fig. 4. Example of recombination process

the convergence is to a local optimum, the fast convergence is desirable if it is to the global optimum.

The discussion about premature convergence goes through other concepts such as exploration, global exploration of search space, and exploitation, local exploitation of the search space EIBEN \& SCHIPPERS (1998); Spears (1992). Thus, we can define premature convergence as little exploration and premature exploitation. Usually, the algorithms that use both strategies apply the global search at the beginning to identify promising regions, and local search later to find the best solution in the good regions.

The GA is an algorithm which begins its execution focusing on exploration strategy, when their genetic diversity is high, and at a certain time it focuses in the exploitation, when a representative portion of individuals have similarities.

To avoid premature convergence to local optimum, it is important to make a good exploration. In the Canonical GA's case, the exploration is done by mutation, with a low probability, and by the recombination of solutions generated by the beginning of the evolutionary process, when the population usually has high genetic diversity. However, this process is slow, considering that several generations are needed to prioritize one of the promising regions.

Allowing the GA to have a better use of its individuals (information mining), we believed that the genetic evolution can be improved without increasing the probability of the premature convergence.

\section{Experiments}

To test the performance of the proposed algorithm (IVFm) and its operators, we chose a benchmark multimodal problem. The Rastrigin's function (Equation 1) is widely used and one of the hardest benchmark because of multimodality and its behavior with many peaks and valleys, featuring several local optimum. Figure 5 shows the function. 


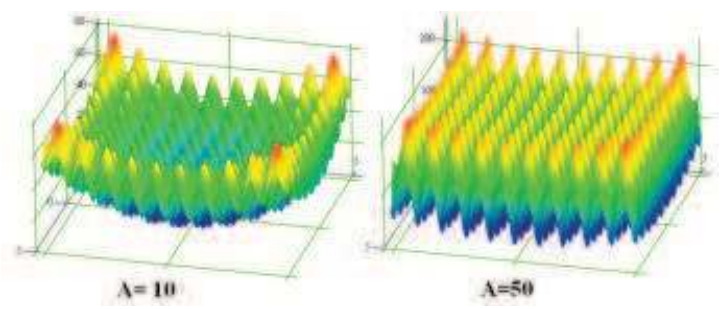

Fig. 5. Bidimensional Rastrigin function with $A=10$ and $A=50$

$$
f(x)=n A+\sum_{i=1}^{n}\left(x_{i}^{2}-A \cos \left(2 \pi x_{i}\right)\right) ; \forall i \in[1 . . n], x_{i} \in[-5.12,5.12]
$$

Given the function, the objective is to minimize yet still respecting the restrictions: $-5.12 \leq$ $X_{i} \leq 5.12$ and values with four decimal places. Considering the restrictions, the minimum point of the function is reached when $\mathrm{Xi}=0$. At this point the solution is global optimum, generating a $f\left(X_{i}\right)=0$.

This work presents 3 experiments. In the first experiment, the objective is to examine the efficacy of the IVFm to minimize (Scenario 1.1) and its behavior when the parameter $\mathrm{A}$ is changed (Scenario 1.2). In the second one, the aim is to analyze the behavior of IVFm when the number of individuals is changed (genetic diversity) in the population. Hence, scenario 2.1 was created with 50 individuals; scenario 2.2 with 10 individuals, and 2.3 with 5 individuals. In the third experiment, the impact of the dimensions increase (variable) is analyzed in IVFm. Scenario 3.1 runs with 2 variables, and scenario 3.2 with 10 variables.

Table 1 shows the configurations of the experiments 1, 2 and 3 . For better visualization, some acronyms were used. Like MP for mutation probability, CP for crossover probability, NuG for number of genes, GO for global optimum, DGM for division of genetic material, SC for stopping criterion, $\mathrm{G}$ for generations and NuIndiv for number of IVFm's individuals. The CP is 0.65 and $\mathrm{MP}$ is 0.01 for all experiments.

\subsection{Experimental results}

The experiments aim to measure the efficiency - the capacity to accelerate the GA's evolution - and efficacy - the capacity to reach the global optimum. In this work, the efficiency is measured by the average of NG and efficacy is analyzed by $\mathrm{NGO} \%$, success rate.

Figures 6, 7 and 8 show, respectively, the results of experiments 1, 2 and 3 of algorithms GA, IVFm/AR (AR), IVFm/EAR-P (EAR-P), IVFm/EAR-T (EAR-T) and IVFm/EAR-N (EAR-N).

\subsection{Analysis of results}

Analyzing the results of scenario 1.1, Table 4 and Figure 7, about the efficacy, we can identify a great performance of the IVFm and its operators because all operators reached the global optimum on all the 20 executions. On the other hand, the GA without the aid of the IVFm did not obtain good results, the canonical GA reached the global optimum on only 4 of the 20 executions (20\%). On Scenario 1.2 the function parameter A was increased, characterizing deepest valleys. Nevertheless, the IVFm and its operators continued with excellent results, $100 \%$ of efficacy. Already the GA presents on average performance with $50 \%$ of efficiency. Analyzing the efficiency on experiment 1 - scenario 1.1, the EAR-N has the best result, followed by EAR-T, EAR-P and AR. Scenario 1.2 identifies a small efficiency loss of the IVFm. The GA without IVFm presents a lower efficiency, even if considering only the average of executions on which the global optimum was reached. 


\begin{tabular}{|c|c|c|c|c|c|c|c|}
\hline \multicolumn{8}{|c|}{ Experiment 1} \\
\hline Scenario & \multicolumn{3}{|c|}{ GA } & \multicolumn{2}{|c|}{ IVFm } & \multicolumn{2}{|c|}{ Function } \\
\hline 1.1 & Pop & $\mathrm{NuG}$ & SC & DGM & NuIndiv & Variables & A \\
\hline & 50 & 34 & $\begin{array}{l}\mathrm{GO} \text { or } \\
250 \mathrm{G}\end{array}$ & 4 & 45 & 2 & 10 \\
\hline 1.2 & Pop & NuG & SC & DGM & NuIndiv & Variables & A \\
\hline & 50 & 34 & $\begin{array}{l}\mathrm{GO} \text { or } \\
250 \mathrm{G}\end{array}$ & 4 & 45 & 2 & 50 \\
\hline \multicolumn{8}{|c|}{ Experiment 2} \\
\hline Scenario & \multicolumn{3}{|c|}{ GA } & \multicolumn{2}{|c|}{ IVFm } & \multicolumn{2}{|c|}{ Function } \\
\hline 2.1 & Pop & $\mathrm{NuG}$ & SC & DGM & NuIndiv & Variables & $\mathbf{A}$ \\
\hline & 30 & 34 & $\begin{array}{l}\mathrm{GO} \text { or } \\
250 \mathrm{G}\end{array}$ & 2 & 27 & 2 & 10 \\
\hline 2.2 & Pop & NuG & SC & DGM & NuIndiv & Variables & A \\
\hline & 10 & 34 & $\begin{array}{l}\mathrm{GO} \text { or } \\
250 \mathrm{G}\end{array}$ & 2 & 9 & 2 & 10 \\
\hline 2.3 & Pop & $\mathrm{NuG}$ & SC & DGM & NuIndiv & Variables & $\mathbf{A}$ \\
\hline & 5 & 34 & $\begin{array}{l}\mathrm{GO} \text { or } \\
250 \mathrm{G}\end{array}$ & 2 & 4 & 2 & 10 \\
\hline \multicolumn{8}{|c|}{ Experiment 3} \\
\hline Scenario & \multicolumn{3}{|c|}{ GA } & \multicolumn{2}{|c|}{ IVFm } & \multicolumn{2}{|c|}{ Function } \\
\hline 3.1 & Pop & $\mathrm{NuG}$ & SC & DGM & NuIndiv & Variables & $\mathbf{A}$ \\
\hline & 50 & 34 & $\begin{array}{l}\mathrm{GO} \text { or } \\
250 \mathrm{G}\end{array}$ & 4 & 45 & 2 & 10 \\
\hline 3.2 & Pop & $\mathrm{NuG}$ & SC & DGM & NuIndiv & Variables & A \\
\hline & 50 & 170 & $\begin{array}{l}\text { GO or } \\
250 \mathrm{G}\end{array}$ & 10 & 45 & 10 & 10 \\
\hline
\end{tabular}

Table 1. Experiments configuration

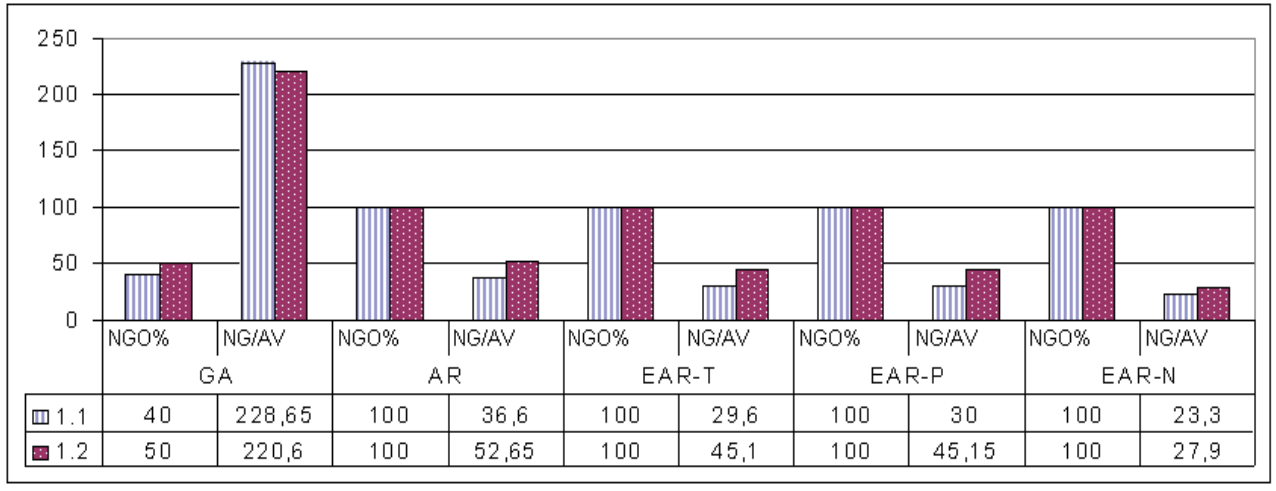

Fig. 6. Experiment 1 results

On experiment 2 we can measure the impact of the reduction of individuals (genetic diversity) on the proposed algorithm. On scenario 2.1 with 30 individuals, all operators of IVFm showed $100 \%$ of efficacy and the GA $50 \%$. On scenario 2.2 with 10 individuals, we can observe a loss 


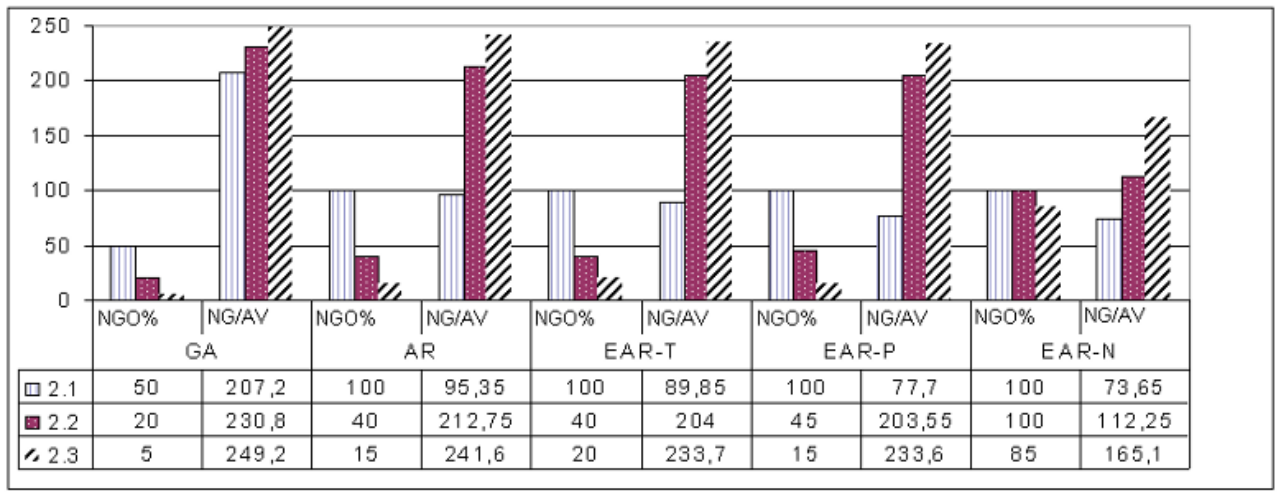

Fig. 7. Experiment 2 results

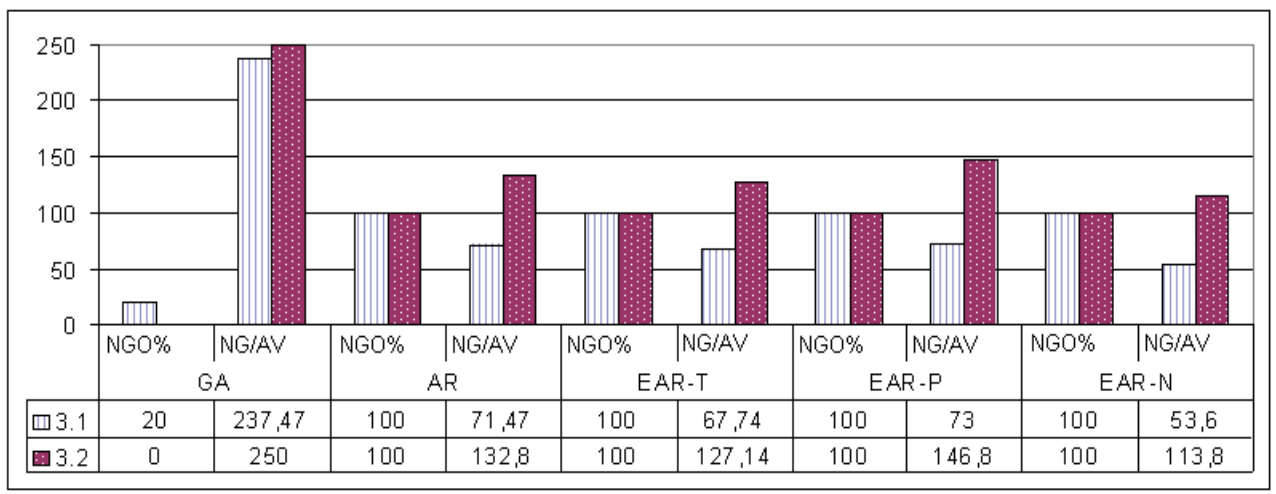

Fig. 8. Experiment 3 results

of performance by the EAR-T (40\%), EAR-P (40\%) and AR (40\%). The EAR-N maintained an excellent efficacy of $100 \%$ and GA fell to $20 \%$. On scenario 2.3 with only 5 individuals, we can prove the thesis that the operators EAR-P, EAR-T and AR, besides the canonical GA, suffer an impact with the reduction of genetic material, because the success rates were $20 \%, 15 \%, 15 \%$ and $5 \%$ respectively. The EAR-N also showed reduction of efficiency on scenario 2.3 , though returned surprising result of efficiency $(85 \%)$, as even with very low genetic diversity.

Besides the direct dependence of the IVFm efficacy with the number of individuals, experiment 2 shows the contribution of EAP to the GA on scenarios with a few genetic material.

Finally, on experiment 3 we can identify the behavior of algorithms when we increased the problem dimension, hence the complexity. On scenario 3.1 with 2 dimensions, variables $X$ and $Y$, the IVFm showed, once again, 100\% of efficacy. On the other hand, the GA without the IVFm showed only $20 \%$ of efficacy. Looking at the average of generations to reach the global optimum (NG/AV), we can identify a better efficiency of EAR-N (53.6), followed by EAR-T (67.73), AR (71.47) and EAR -P (73). On scenario 3.2 with 10 dimensions, the IVFm keeps the $100 \%$ of efficacy, but we can observe a decrease in efficiency because of the NG/AV: 113.8 (EAR-N), 127.13 (EAR-T), 132.8 (AR) and 146.8 (EAR-P). Maintaining the effectiveness of $100 \%$ on scenario 3.2 , IVFm showed its robustness to Rastrigin multidimensional problem. 
On this scenario, the GA presents its worst performance, because it did not reach the global optimum at any time, demonstrating the difficulty to evolve on highly multimodal and multidimensional problems.

\section{Conclusion}

Some papers RAJAN et al. (2002); SINGH \& DEB (2006); YANG \& DOUGLAS (1998) propose new algorithms that, independently, have better results than the GA. On some benchmarks, however, this study suggests a joining algorithm (IVFm) that allows a better use of information generated by the GA or by the IVFm.

Tests with the benchmark Rastrigin were executed to measure the effectiveness and efficiency of the proposed IVFmGA. The established scenarios were divided in to 3 experiments with different purposes. In the first one, the capacity of the IVFm was measured to reach the global optimum in a multimodal context. The IVFm proved to be effective and efficient, because it reached the global optimum in few generations, even with the increase of the function parameter A that increased a larger complexity to the problem.

In the second experiment, some scenarios were set up to analyze the impact of the individuals' reduction in the population. This reduction decreases the material and the genetic diversity, which is usually very damaging to the evolutionary algorithms. With 30 individuals, scenario 2.1, IVFm was $100 \%$ effective, already with 10 and 5 the operators EAR-T, EAR-P and AR suffered a reduction of effectiveness and efficiency. The operator EAR-N obtained better results with $100 \%$ in scenarios 2.1 and 2.2 and $85 \%$ in scenario 2.3 . These are considered excellent performances because although it only had 5 individuals, it was capable of reaching the global optimum in $85 \%$ of the cases.

In the experiment 3, the impact of the dimension increase in the algorithms was measured. The IVFm had $100 \%$ of efficacy and a low efficiency reduction when the dimension increased to 10. This result shows a robustness of the IVFm on dimensionality. The operators EAR-T, EAR-P and AR had a good efficacy and robustness too.

Analyzing all tests done, we can observe that the IVFm was able to considerably increase the efficacy and efficiency of GA.

Among the IVFm operators, the EAR-N was more effective and efficient than the others, followed by EAR-T, EAR-P and AR. The good performance of the EAR-N is justified by the way that it balances the exploration of the search area, generating new information, and exploitation of promising areas, promoted by the recombination process.

Given the tests presented, we can notice that the IVFm has a great potential to help various kinds of binary-coded evolutionary algorithms. Therefore, it is suggested as future work the joining of the IVFm in different evolutionary algorithms for different types of applications.

\section{References}

CHAINATE, W. T. \& PONGCHAROEN, P. (2007). A new heuristic for improving the performance of genetic algorithm, Academy of Science, Engineering and Technology 21(1): 217-220.

EIBEN, A. E. \& SCHIPPERS, C. A. (1998). On evolutionary exploration and exploitation, Fundamenta Informaticae 35: 35-50.

FERREIRA, C. (2001a). Gene expression programming: A new adaptive algorithm for solving problems, Complex Systems 13(2): 87-129.

FERREIRA, C. (2001b). Gene expression programming in problem solving, Tutorial, Universidade dos Açores, Portugal.

URL: http://www.propesq.ufpe.br/anais/anais.htm 
GEN, M. \& CHENG, R. (1997). Genetic Algorithms and Engineering Design, EDA.

Goldberg, D. E. (1989). Genetic Algorithms in Search, Optimization, and Machine Learning, Addison-Wesley Professional.

Jung, S. H. (2003). Queen-bee evolution for genetic algorithms, Electronics Letters 39(6): 575-576.

MAHFOUD, S. (1992). Crowding and preselection revisited, in R. Manner \& B. Manderick (eds), Parallel Problem Solving from Nature 2, Amsterdan, pp. 27-36.

MAHFOUD, S. (1995). Niching methods for genetic algorithms, Tech report, Department of General Engineering, University of Illinois at Urbana-Champaign.

MICHALEWICZ, Z. (1996). Genetic algorithms + data structures = evolution programs, Springer-Verlag, Berlin.

MUSIL, M., WILMUT, M. J. \& CHAPMAN, R. (1999). A hybrid simplex genetic algorithm for estimating geoacoustic parameters using matched-field inversion, IEEE Journal of Oceanic Engineering 24(3): 358-369.

PARK, J.-B., PARK, Y.M. abd WON, J. \& LEE, K. (2000). An improved genetic algorithm for generation expansion planning, Power Systems 15(1): 916-922.

RAJAN, C., MOHAN, M. \& MANIVANNAN, K. (2002). Improved genetic algorithm solution to unit commitment problem, Transmission and Distribution Conference and Exhibition, IEEE/PES, Asia Pacific, pp. 255-260.

RODRIGUES, E. (2003). Genetic programming in the optimization of digital circuits (in portuguese), IV Encontro Nacional de Inteligência Artificial, Campinas, Brazil.

RONG-LONG, W. \& KOZO, O. (2005). Solving facility layout problem using an improved genetic algorithm, Fundamentals of Electronics, Communications and Computer Sciences $\mathrm{E} 88-\mathrm{A}(2)$ : 606-610.

RUTTKAY, Z., EIBEN, A. \& RAUE, P. (1995). Improving the performances of gas on a ga-hard csp, Workshop on Studying and Solving Really Hard Problems, Cassis, França, pp. 157-171.

SHIMODAIRA, H. (2002). An empirical performance comparison of niching methods for genetic algorithms, IEICE Trans Inf Syst E85-D(11): 1872-1880.

SINGH, G. \& DEB, K. (2006). Comparison of multi-modal optimization algorithms based on evolutionary algorithms, 8th annual conference on Genetic and evolutionary computation, Seattle, USA, pp. 1305-1312.

Spears, W. M. (1992). Crossover or mutation?, Foundations of Genetic Algorithms 2, Morgan Kaufmann, pp. 221-237.

SULTAN, A. B. M., MAHMUD, R. \& S., S. M. (2007). Reducing premature convergence problem through numbers structuring in genetic algorithm, International Journal of Computer Science and Network Security 7(4): 215-217.

TACKETT, W. A. \& CARMI, A. (1994). The unique implications of brood selection for genetic programming, IEEE World Congress on Computational Intelligence, Orlando, FL, USA, pp. 160-165.

WU, A. S., YU, H., JIN, S., LIN, K. C. \& SCHIAVONE, G. (2004). An incremental genetic algorithm approach to multiprocessor scheduling, Parallel and Distributed Systems 15(9): 824-834.

YANG, R. \& DOUGLAS, I. (1998). Simple genetic algorithm with local tuning: Eficient global optimizing technique, J. Optim. Theory Appl. 98(2): 449-465.

YEN, J., LIAO, J. C., LEE, B. \& RANDOLPH, D. (1998). A hybrid approach to modeling metabolic systems using a genetic algorithm and simplex method, IEEE Trans. on Syst., Man, and Cybern. 28(2): 173-191. 


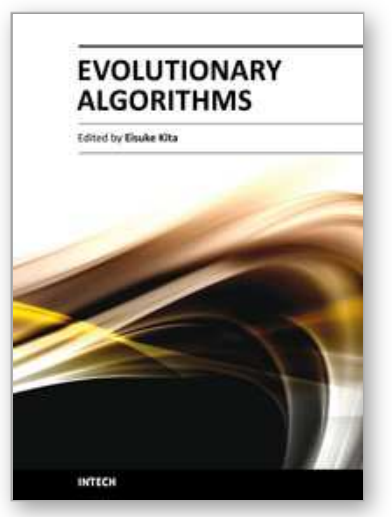

\author{
Evolutionary Algorithms \\ Edited by Prof. Eisuke Kita
}

ISBN 978-953-307-171-8

Hard cover, 584 pages

Publisher InTech

Published online 26, April, 2011

Published in print edition April, 2011

Evolutionary algorithms are successively applied to wide optimization problems in the engineering, marketing, operations research, and social science, such as include scheduling, genetics, material selection, structural design and so on. Apart from mathematical optimization problems, evolutionary algorithms have also been used as an experimental framework within biological evolution and natural selection in the field of artificial life.

\title{
How to reference
}

In order to correctly reference this scholarly work, feel free to copy and paste the following:

Celso G. Camilo-Junior and Keiji Yamanaka (2011). In Vitro Fertilization Genetic Algorithm, Evolutionary Algorithms, Prof. Eisuke Kita (Ed.), ISBN: 978-953-307-171-8, InTech, Available from: http://www.intechopen.com/books/evolutionary-algorithms/in-vitro-fertilization-genetic-algorithm

\section{INTECH}

open science | open minds

\section{InTech Europe}

University Campus STeP Ri

Slavka Krautzeka 83/A

51000 Rijeka, Croatia

Phone: +385 (51) 770447

Fax: +385 (51) 686166

www.intechopen.com

\section{InTech China}

Unit 405, Office Block, Hotel Equatorial Shanghai

No.65, Yan An Road (West), Shanghai, 200040, China 中国上海市延安西路65号上海国际贵都大饭店办公楼 405 单元

Phone: +86-21-62489820

Fax: $+86-21-62489821$ 
(C) 2011 The Author(s). Licensee IntechOpen. This chapter is distributed under the terms of the Creative Commons Attribution-NonCommercialShareAlike-3.0 License, which permits use, distribution and reproduction for non-commercial purposes, provided the original is properly cited and derivative works building on this content are distributed under the same license. 